\title{
Alte Substanzen, neue Erkenntnisse: Immunmodulatoren und Immunsuppressiva beim kutanen Lupus erythematodes
}

Known Substances, New Findings: Immunomodulatory and Immunosuppressives

in the Treatment of Cutaneous Lupus Erythematosus

\section{Zusammenfassung}

Für die Therapieentscheidung des kutanen Lupus erythematodes (LE) ist die möglichst genaue Einordnung des Krankheitsbildes in das Spektrum der verschiedenen kutanen Varianten erforderlich. Am häufigsten sind der chronisch-diskoide LE (CDLE) und der subakut-kutane LE (SCLE). Neben der kutanen Ausdehnung ist das Vorhandensein einer extrakutanen Beteiligung für die Therapieentscheidung wichtig. Standardtherapeutika des kutanen LE sind Antimalariamittel (Chloroquin, Hydroxychloroquin) allein oder in Kombination mit systemischen Kortikosteroiden. Auch Arthralgien/Arthritiden werden durch diese Therapie günstig beeinflusst. Bei anderen, extrakutanen Manifestationen wird dem Azathioprin der Vorzug gegeben. In 80-90\% der Patienten erzielt man mit der Standardtherapie ein gutes therapeutisches Ansprechen. Alternativ hierzu können, bei Therapieversagern oder wenn die Standardtherapie aus anderen Gründen nicht einsetzbar ist, orale Retinoide, Thalidomid, Methotrexat oder Diaminodiphenylsulfon versucht werden. In neueren, kasuistischen Mitteilungen wird über die erfolgreiche lokale Anwendung von Tacrolimus und den systemischen Einsatz von Mycophenolatmofetil beim kutanen LE berichtet.

\section{Abstract}

For therapy of cutaneous lupus erythematosus the precise classification of the clinical subset is necessary. The most frequently found LE subsets are chronic discoid LE (CDLE) and subacute cutaneous LE (SCLE). Extension of the cutaneous lesions and presence of visceral involvement are criteria for therapy choice. Basic therapeutics for cutaneous LE are antimalarials (chloroquine, hydroxychloroquine) alone or in combination with systemic corticosteroids. For visceral involvement others than arthralgia/arthritis azathioprin is the first line therapy. In $80-90 \%$ of the patients disease can be controlled by these standard therapies. In the remaining cases oral retinoids, thalidomide, methotrexate or diaminodiphenylsulfon can be tried. Recently, a few case reports have been published on a good therapeutic effect of local tacrolimus and systemic mycophenolate mofetil in cutaneous LE.

\section{Einleitung}

Der systemische Lupus erythematodes (SLE) ist eine der häufigsten Autoimmunkrankheiten. Prävalenzraten variieren weltweit zwischen 14 und 50/100 000 in der Bevölkerung [24]. Zum kutanen Lupus erythematodes gibt es demgegenüber keine gut dokumentierten, epidemiologischen Studien. Wahrscheinlich ist der kutane LE jedoch 2-3-mal häufiger als der SLE. Die Prognose des SLE wird durch die Schwere und die Ausdehnung der inneren
Organbeteiligung bestimmt. Die Mehrzahl von ungünstigen Krankheitsverläufen ist auf Manifestationen an den Nieren und dem zentralen Nervensystem zurückzuführen sowie verschiedenen, während der Behandlung auftretenden Infektionen. Die 10-Jahres-Überlebensrate des SLE liegt heute bei rund $80 \%$, bedingt durch die Behandlung mit systemischen Kortikosteroiden und Immunsuppressiva [14,18]. Die Prognose des kutanen LE ist als günstiger einzustufen als die des SLE. Schwere Krankheitsverläufe mit letalem Ausgang sind eine Rarität. Allerdings liegen zur 
Prognose des kutanen LE keine prospektiven Studien an größeren Patientenkollektiven vor.

Als Dermatologe ist man in erster Linie mit Patienten konfrontiert, die an einem kutanen LE erkrankt sind. Die häufigsten, kutanen Varianten des LE sind der chronisch-diskoide LE (CDLE) und der subakut-kutane LE (SCLE). Andere seltenere Varianten sind der LE profundus/panniculitis, LE tumidus, LE hypertrophicus, bullöser LE, Urticaria vasculitis u.a. Das Schmetterlingserythem und/oder makulöse Exantheme sind charakteristische Hautbefunde des SLE. CDLE und SCLE können in jedem Lebensalter auftreten. Die meisten Patienten befinden sich bei Krankheitsbeginn jedoch zwischen dem 30. und 50. Lebensjahr. Frauen sind deutlich häufiger betroffen mit einem Überwiegen von $3: 1$ beim CDLE und 3-6:1 beim SCLE. Neben dem klassischen, LEspezifischen Hautbefund zeigen viele Patienten LE-unspezifische Hautbefunde wie akrolokalisierte Vaskulitis (4-30\%), Livedo racemosa $(22-35 \%)$ und diffuser Haarausfall $(38-78 \%)[3,36,54]$.

Zeichen einer beginnenden extrakutanen Manifestation wie Arthralgien und/oder geringe Proteinurie können bei CDLE-Patienten in $14-27 \%$ und bei SCLE-Patienten in $67-70 \%$ der Fälle vorhanden sein $[50,54,60]$. Ungefähr $5-10 \%$ der Patienten mit CDLE entwickeln über einen mehrjährigen (> 5 Jahre) Krankheitsverlauf einen Übergang in einen SLE $[23,33,35]$. Von den SCLE-Patienten entwickeln 50-60\% extrakutane Manifestationen nach mehreren Jahren $[10,28,50]$.

Obgleich CDLE und SCLE aufgrund des klinischen und histologischen Befundes einfach zu diagnostizieren sind, ist es im Einzelfall schwierig zu entscheiden, welcher Patient einen schweren Krankheitsverlauf nehmen wird und eine systemische Variante entwickelt. Die Kriterien der American Rheumatism Association (ARA) sind wenig geeignet für den Dermatologen [52]. Nach diesen Kriterien werden bereits 20\% der CDLE- und 30-50\% der SCLE-Patienten als SLE klassifiziert, die sich jedoch hinsichtlich Krankheitsverlauf und Prognose von SLE-Patienten mit schwerer Nierenbeteiligung oder ZNS-Beteiligung eindeutig unterscheiden $[3,43]$.

Es ist Aufgabe des Dermatologen, diejenigen Patienten zu erkennen, die ein erhöhtes Risiko für einen schweren Krankheitsverlauf haben und einen SLE entwickeln. In den letzten Jahren sind verschiedene Versuche unternommen worden, sich mit dieser Problematik näher auseinanderzusetzen. Zum einen hat man versucht Kriterien zur Bestimmung der Krankheitsaktivität, die für den SLE entwickelt wurden, auf Patienten mit kutanem LE anzuwenden. Zum anderen erfolgten vergleichende Untersuchungen zwischen Patienten mit kutanem LE und SLE, um Risikofaktoren für den Übergang des kutanen LE in den SLE zu definieren.

Einige, wenige Studien sind publiziert, in denen für den SLE gut etablierte Evaluationskriterien zur Bestimmung der Krankheitsaktivität auf den kutanen LE übertragen wurden. In einer Studie wurden 176 Patienten mit kutanem LE anhand des Systemic Lupus Activity Measure (SLAM) evaluiert und über einen durchschnittlichen Zeitraum von 9 Jahren nachbeobachtet. Wesentliche Ergebnisse dieser Studie waren, dass nur $2 \%$ der Patienten einen sehr aktiven Krankheitsprozess im Sinne dieser Aktivitäts- skala hatten [44]. Der Nachteil von SLAM besteht darin, dass zwischen einzelnen kutanen LE-Varianten nicht unterschieden wird. Patienten mit Schmetterlingserythem, CDLE, Lupus profundus und bullösem LE werden gleichrangig gewertet. Ein anderer Aktivitätsscore, der Systemic Lupus Erythematosus Disease Activity Index (SLEDAI), zeigte, dass SLE-Patienten mit unspezifischen Hautläsionen eine höhere Krankheitsaktivität haben, als solche mit LE-spezifischen Hautmanifestationen. Dadurch wird die Hypothese unterstützt, dass die letzte Gruppe, einschließlich CDLE/ SCLE-Patienten, eine günstigere Prognose hat [64].

Aus dermatologischer Sicht ist es erforderlich Kriterien zu definieren, die es ermöglichen, solche Patienten mit kutanem LE zu erkennen, die ein erhöhtes Risiko haben, einen schweren Krankheitsverlauf mit Übergang in SLE zu entwickeln. In einer prospektiven Multizenterstudie an insgesamt 296 LE-Patienten wurde eine große Anzahl von klinischen und serologischen Parametern im Hinblick auf die Aussagekraft als mögliche Risikofaktoren für den kutanen LE untersucht. Wesentliche Ergebnisse dieser Studie waren, dass geringe Zeichen einer Nierenbeteiligung (Proteinurie, Hämaturie) die höchste statistische Signifikanz hatten, um zwischen Patienten mit kutanem LE (CDLE/ SCLE) und SLE zu unterscheiden, gefolgt von positivem ANA-Titer ( $\geq 1: 320$ ), länger als 3 Monate bestehenden Arthralgien/Arthritiden und BSG-Erhöhung. Demgegenüber waren ein nur gering erhöhter ANA-Titer sowie der Nachweis von anti-ds-DNA-Antikörpern nicht aussagekräftig genug, um zwischen diesen beiden LEKollektiven zu unterscheiden [53]. Anti-ds-DNA-Antikörper sind hoch sensitiv für die Diagnose LE. Allerdings werden mit den heute zur Verfügung stehenden ELISA-Testkits nicht nur die hoch affinen Antikörper vom IgG-Typ, sondern ebenso weniger spezifische, niedrig affine Antikörper vom IgM-Typ nachgewiesen [16]. Dadurch fällt auch bei Patienten mit kutanem LE dieser Test häufig positiv aus.

Für die Therapieentscheidung ist wesentlich, die Einordnung des klinischen Befundes unterstützt durch Histologie, Immunhistologie und immunserologisches Profil in eine der bekannten Untergruppen des kutanen LE vorzunehmen. Die Wahl der individuellen Therapie richtet sich neben der Ausdehnung der Hautbeteiligung nach dem Vorhandensein milder, klinischer Zeichen einer extrakutanen Manifestation der Erkrankung (Abb.1). Es ist die Aufgabe des Dermatologen, der wegen der Hautbeteiligung in aller Regel als erster von den Patienten konsultiert wird, im Individualfall eine möglichst exakte Einordnung des kutanen LE und eine Abgrenzung zum SLE mit schwerer extrakutaner Manifestation vorzunehmen.

\section{Systemische Standardtherapie}

Nach wie vor gelten Antimalariamittel allein oder in Kombination mit systemischen Kortikosteroiden als Standardtherapie des kutan-disseminierten LE. LE-assoziierte Arthralgien/Arthritiden werden durch diese Therapie ebenfalls effizient behandelt. Chloroquin (Dosis: $250 \mathrm{mg} / \mathrm{d}$ ) bzw. Hydroxychloroquin (Dosis: $200-400 \mathrm{mg} / \mathrm{d}$ ) zeigen ihre volle Wirkung erst nach 2-4 Monaten Behandlungszeit, so dass eine Kombination mit systemischen Kortikosteroiden (initial: $20-30 \mathrm{mg} / \mathrm{d}$ ) sinnvoll ist, um den Krankheitsverlauf abzukürzen. 


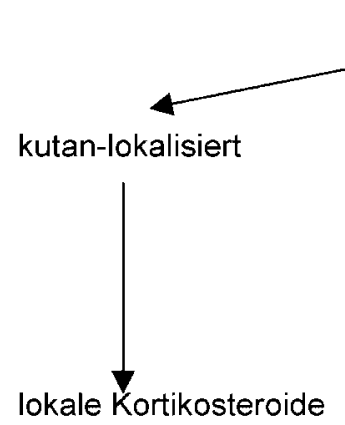

CDLE/SCLE

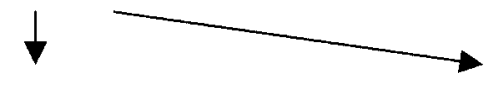

kutan-disseminiert ohne oder mit Arthralgien/Arthritis

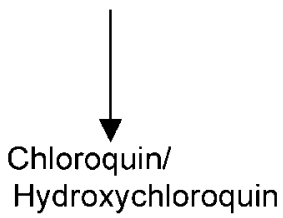

allein oder in Kombination mit systemischen Kortikosteroiden
Abb. 1 Standardtherapie des CDLE und des SCLE. kutan-disseminiert mit extrakutaner Manifestation (außer Arthralgien/Arthritis)

Azathioprin in Kombination mit systemischen Kortikosteroiden

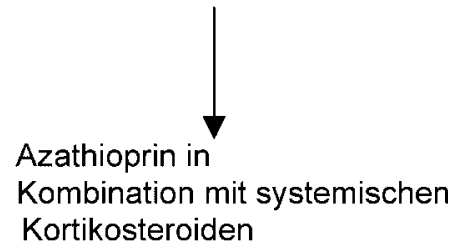

Eine neuere Studie hat gezeigt, dass die Wirksamkeit von Antimalariamittel bei Patienten mit CDLE oder SCLE davon abhängt, ob der Patient Raucher oder Nichtraucher ist. In einer vergleichenden Studie waren Antimalariamittel bei Rauchern signifikant weniger wirksam als bei Nichtrauchern [27]. Eine mögliche Erklärung ist, dass Zigarettenrauch die Aufnahme von Antimalariamitteln in Lysosomen inhibiert und somit die pharmakologische Wirkung vermindert [62].

Antimalariamittel greifen an mehreren Stellen in den kutanen Krankheitsprozess beim LE ein. Sie legen sich in den Keratinozyten wie ein physikalischer Schutzschild um den Zellkern. Sie inhibieren die UV-induzierte Aktivität von Phospholipase A und C und wirken somit über eine verminderte Prostaglandinsynthese antiinflammatorisch. Antimalariamittel werden in Lysosomen aufgenommen, stabilisieren diese und verhindern die Freisetzung von Proteasen. Des Weiteren inhibieren sie die zellmediierte Zytotoxizität [62].

Antimalariamittel können reversible und irreversible Augenschäden verursachen. Daher sind regelmäßige, augenärztliche Kontrollen zu empfehlen. Die irreversible Retinopathie ist insgesamt seltener unter Hydroxychloroquin- als unter Chloroquintherapie. Eine immer wieder kontrovers diskutierte Frage ist, ob die Retinopathie nach einer Tageshöchstdosis oder eher nach einer kumulativen Gesamtdosis vermehrt auftritt. Die Gesamtdosis von Chloroquin sollte $500 \mathrm{~g}$ nicht überschreiten; erst ab einer Gesamtdosis von $250 \mathrm{~g}$ sind Retinopathien festgestellt worden. Wenn die Tagesdosis von Hydroxychloroquin nicht mehr als $6,5 \mathrm{mg} / \mathrm{kg}$ KG beträgt, besteht auch bei Langzeittherapie nur ein minimales Risiko für die Entwicklung einer Retinopathie [29]. Weitere Nebenwirkungen der Antimalariamittel sind Nausea, Erbrechen, Pruritus, Leukopenie, Thrombozytopenie, Nervosität, Muskelschwäche, neurologische Störungen (Paresen, Pyschosen), Phototoxizität, Exantheme, Aufhellung der Haare u.a. [59].

Spricht das Krankheitsbild nicht auf die genannte Therapie mit Antimalariamitteln an oder liegen andere extrakutane Manifestationen vor, ist eine Therapie mit Azathioprin (Dosis: $1 \mathrm{mg} / \mathrm{kg}$ $\mathrm{KG} / \mathrm{d}$ ) in Kombination mit systemischen Kortikosteroiden sinnvoll. Meist ist eine Langzeittherapie erforderlich. Ein Auslassversuch sollte erst nach mindestens 6 Monaten klinischer Erscheinungsfreiheit erfolgen. Der Wirkungseintritt ist nach etwa 2 Mo- naten zu erwarten. Insbesondere in Fällen eines SLE mit Nierenbeteiligung konnte gezeigt werden, dass Azathioprin als Langzeittherapie, der seit längerem für diese Indikation empfohlenen Cyclophosphamid-Pulstherapie, gleichwertig ist [20,38].

Die häufigsten Nebenwirkungen von Azathioprin sind Myelosuppression, opportunistische Infektionen und Leberzellveränderungen. Die Myelosuppression kann sich rasch in der Einleitungsphase der Therapie entwickeln. Azathioprin wird abgebaut u. a. durch Thiopurinmethyltransferase (TPMT). Für dieses Enzym besteht ein genetischer Polymorphismus, wodurch bei etwa $10 \%$ der Patienten eine TPMT-Defizienz bzw. eine geringe Enzymaktivität vorliegt [37]. Dadurch kommt es bei diesen Patienten zu einem Anstieg von zytotoxischen Thioguanin-Nukleotiden, was mit einer erhöhten Rate von Leukopenien korreliert [12]. Eine neuere Arbeit konnte allerdings zeigen, dass eine normale TPMT-Aktivität das Auftreten von einer Myelosuppression bei SLE-Patienten nicht ausschließt [37]. Daher sind in der Einleitungsphase einer Azathioprin-Therapie bei jedem Patienten Blutbildkontrollen in den ersten 8 Wochen nach Therapiebeginn wöchentlich und anschließend in Intervallen von 4 Wochen $\mathrm{zu}$ empfehlen.

In 80 -90\% der Patienten mit kutanem LE lässt sich das Krankheitsbild unter der genannten Standardtherapie effektiv kontrollieren. Für Fälle, in denen keine Remission des Krankheitsbildes mit der genannten Standardtherapie erreicht werden kann, steht eine Reihe von therapeutischen Alternativen zur Verfügung.

\section{Etablierte therapeutische Alternativen}

Beim kutanen LE sind viele Medikamente als therapeutische Alternativen versucht worden (Tab.1). Kontrollierte Studien liegen jedoch nur für wenige Medikamente vor. Im Folgenden wird eine Auswahl von diesen Medikamenten näher vorgestellt, für die umfangreicheres Datenmaterial über ihre Anwendung beim kutanen LE vorliegt bzw. die, aufgrund eigener Erfahrungen, in den engeren Kreis der empfehlenswerten, therapeutischen Alternativen zu setzen sind (Tab. 2 ).

\section{Orale Retinoide}

Retinoide sind seit den 80er-Jahren etablierte Therapeutika beim kutanen LE. Sowohl für das Etretinat als auch das Acitretin konnte in kontrollierten Studien gezeigt werden, dass die Wirksam- 
Tab. 1 Therapeutische Alternativen beim kutanen LE

\begin{tabular}{|c|c|c|c|}
\hline \multicolumn{4}{|c|}{ Acitretin/Etretinat/Isotretinoin } \\
\hline \multicolumn{4}{|c|}{ Thalidomid } \\
\hline \multicolumn{4}{|l|}{ Methotrexat } \\
\hline \multicolumn{4}{|c|}{ Diaminodiphenylsulfon } \\
\hline \multicolumn{4}{|c|}{ Clofazimin } \\
\hline \multicolumn{4}{|l|}{ Auranofin } \\
\hline \multicolumn{4}{|l|}{ Phenytoin } \\
\hline \multicolumn{4}{|c|}{ Sulfazalazin } \\
\hline \multicolumn{4}{|c|}{ Danazol (bei Frauen) } \\
\hline \multicolumn{4}{|c|}{ Vitamin $\mathrm{E}$} \\
\hline \multicolumn{4}{|l|}{ Betacaroten } \\
\hline \multicolumn{4}{|l|}{ Experimentell: } \\
\hline \multicolumn{4}{|c|}{ Interferon alpha } \\
\hline \multicolumn{4}{|c|}{ Anti-CD-4-Antikörper } \\
\hline \multicolumn{4}{|c|}{ extrakorporale Photopharese } \\
\hline \multicolumn{4}{|c|}{ UVA1-Bestrahlung } \\
\hline \multicolumn{4}{|l|}{ nach [42] } \\
\hline \multicolumn{4}{|c|}{ Tab. 2 Etablierte therapeutische Alternativen beim kutanen LE } \\
\hline & Indikation & Dosis & Wirkungseintritt \\
\hline Acitretin & $\begin{array}{l}\text { CDLE } \\
\text { SCLE } \\
\text { LE hypertrophicus }\end{array}$ & $\begin{array}{l}0,35-0,5 \mathrm{mg} / \mathrm{kg} \\
\mathrm{KG} / \mathrm{d}\end{array}$ & nach 2-3 Wochen \\
\hline Thalidomid & $\begin{array}{l}\text { CDLE } \\
\text { SCLE }\end{array}$ & $50-400 \mathrm{mg} / \mathrm{d}$ & nach 2 Wochen \\
\hline Methotrextat & $\begin{array}{l}\text { CDLE } \\
\text { SCLE } \\
\text { LE profundus }\end{array}$ & $10-25 \mathrm{mg} /$ Woche & nach 2-4 Wochen \\
\hline DADPS & $\begin{array}{l}\text { Urticaria vasculitis } \\
\text { Bullöser LE } \\
\text { SCLE }\end{array}$ & $100-150 \mathrm{mg} / \mathrm{d}$ & nach 2-3 Wochen \\
\hline
\end{tabular}

nach [42]

keit beim LE in etwa der von Antimalariamitteln entspricht $[46,47]$. Sie sind beim CDLE und SCLE wirksam sowie insbesondere beim LE hypertrophicus. In einer doppelblinden, randomisierten Multizenterstudie wurden 28 LE-Patienten mit Acitretin (Dosis: $50 \mathrm{mg} / \mathrm{d}$ ) und 30 LE-Patienten mit Hydroxychloroquin (Dosis: $400 \mathrm{mg} / \mathrm{d}$ ) behandelt. Nach einem Beobachtungszeitraum von 8 Wochen zeigten 50\% der Acitretin-Behandelten und $46 \%$ der Hydroxychloroquin-Behandelten eine deutliche Besserung [47]. Aus der klinischen Erfahrung heraus lässt sich sagen, dass bei einer längerfristigen Therapie und/oder einer Kombination mit niedrigdosierten, systemischen Kortikosteroiden die Ansprechrate noch höher liegt. Isotretinoin wurde in einer Dosis von $80 \mathrm{mg} / \mathrm{d}$ in Einzelfallberichten als wirksam beschrieben [49]. Systemische Retinoide sind allerdings beim LE in aller Regel nur morbostatisch wirksam und Rezidive wurden häufig nach dem Absetzen beobachtet.

Das potenzielle Risiko der Teratogenität von Retinoiden begrenzt den Einsatz. Frauen im gebärfähigen Alter sollten nicht mit diesem Medikament behandelt werden oder nur dann, wenn ein sicherer Konzeptionsschutz vorliegt.

\section{Thalidomid}

In den letzten Jahren konnte gezeigt werden, dass Thalidomid ein effektives Medikament in der Behandlung des LE ist, sowohl des CDLE als auch des SCLE. In einer Dosis von 50-400 mg/d wirkt es innerhalb von wenigen Wochen. Nach einer Behandlungszeit von 8-12 bzw. 16 Wochen zeigte sich bei $80 \%$ bzw. 90\% der Patienten eine Remission des LE [1,6,26,31,41,51]. Auch eine niedrig dosierte Erhaltungstherapie mit $25-50 \mathrm{mg} / \mathrm{d}$ ist als ausreichend wirksam beschrieben $[15,40]$. Thalidomid ist in Deutschland nicht zugelassen. Das Medikament kann allerdings für Einzelfälle direkt vom Hersteller bezogen und im Rahmen eines Heilversuches mit Zustimmung des Patienten eingesetzt werden.

Thalidomid hat immunmodulatorische und antiinflammatorische Eigenschaften, die möglicherweise die Wirkung beim kutanen LE erklären. Thalidomid inhibiert die Leukozytenchemotaxis in Entzündungsreaktionen und verändert die Dichte von TNF- $\alpha$ induzierten Adhäsionsmolekülen an der Oberfläche von Leukozyten. Es erhöht die Produktion von Interleukin-4 und -5 und inhibiert die Produktion von Interleukin-12 und TNF- $\alpha$ durch Monozyten und Makrophagen [9]. Thalidomid hat offenbar beim LE auch Auswirkungen auf messbare, immunologische Parameter. Ein Anstieg der peripheren Lymphozytenzahl sowie ein Absinken von C-reaktivem Protein und anti-ds-DNA-Antikörpern sind berichtet [57].

Thalidomid hat eine Reihe von Nebenwirkungen, die die Anwendung dieses Medikamentes beschränken. Es ist teratogen; die Verordnung bei Frauen im gebärfähigen Alter ist entweder zu unterlassen bzw. darf nur bei sicherem Konzeptionsschutz erfolgen. In annähernd 30\% der Patienten treten periphere Polyneuropathien auf, die bei einem Teil der Patienten irreversibel sind. Das Auftreten einer Polyneuropathie korreliert weder mit der täglichen Dosis noch mit der Dauer der Therapie [40]. Weitere Nebenwirkungen sind u.a. Müdigkeit, Schwindel, Obstipation, Übelkeit, periphere Ödeme und verminderte Libido [55].

\section{Methotrexat}

Methotrexat hat in den letzten Jahren durch sich mehrende Berichte über den erfolgreichen Einsatz beim kutanen LE in gewissem Sinne eine Renaissance in der LE-Therapie erfahren. Verschiedene Arbeitsgruppen haben in Einzelfällen oder an kleineren Fallzahlen berichtet, dass Methotrexat (Dosis: $10-25 \mathrm{mg} / \mathrm{d}$ ) in ansonsten therapierefraktären LE-Fällen wirksam war $[4,7,19,32]$. In einer doppelblinden, plazebokontrollierten Studie mit 41 SLE-Patienten konnte gezeigt werden, das Methotrexat in einer Dosis von $15-20 \mathrm{mg} / \mathrm{d}$ wirksam ist und dabei nicht nur einen günstigen Effekt auf die kutanen LE-Manifestationen hatte, sondern ebenfalls auf die LE-assoziierten Gelenkbeteiligungen [11].

Methotrexat blockiert die DNS-Synthese und damit die Zellproliferation. Ebenso werden die Chemotaxis von neutrophilen Granulozyten sowie die Freisetzung von Zytokinen, wie TNF- $\alpha$, Interleukin-1, -6 und -8 inhibiert, was möglicherweise die Wirksamkeit beim LE erklärt $[2,4]$.

Relativ häufige Nebenwirkungen einer Methotrexattherapie sind Nausea, Vomitus und Abgeschlagenheit. Schwerwiegender sind 
Myelosuppression, Hepatotoxizität und Teratogenität [2]. Regelmäßige Laborkontrollen sind zur Therapieüberwachung unbedingt erforderlich.

\section{Diaminodiphenylsulfon (DADPS)}

DADPS wirkt gut in den LE-Varianten, die mit einer Vaskulitis einhergehen [39]. Ebenfalls günstig wird der SCLE durch DADPS beeinflusst $[17,25,56]$. Gute therapeutische Ergebnisse sind auch für den bullösen LE berichtet [8]. Die Tagesdosis ist in der Regel 100-150 mg DADPS. Weniger gut wirksam ist DADPS beim CDLE, die Ansprechrate liegt nur bei etwa 50\% [13,34].

Der Wirkungsmechanismus von DADPS bei den genannten LEVarianten ist nicht hinreichend geklärt. Ein Effekt ist, dass DADPS und stärker noch sein Hydroxylamin-Metabolit die 5-Lipooxygenaseaktivität polymorphonukleärer Leukozyten inhibieren und damit die Chemotaxis dieser Zellen [61].

Die häufigsten Nebenwirkungen von DADPS sind Kopfschmerzen und Nausea, die meist in den ersten Wochen auftreten und sich bei weiterer Therapie spontan zurückbilden. Dosisabhängige Nebenwirkungen sind die Methämoglobinbildung und eine hämolytische Anämie. Letztere tritt bei Patienten mit hereditärem Glukose-6-Phosphatasemangel auf. Bei risikoverdächtigen Patienten (Bewohner des östlichen Mittelmeerraumes, Afrikaner, Chinesen) sollte daher eine Bestimmung des Enzyms vor Therapiebeginn mit DADPS erfolgen. Arzneimittelexantheme und Polyneuropathien sind ebenso mögliche Nebenwirkungen der DADPS-Therapie [61]. Eine bessere Verträglichkeit von DADPS soll durch die gleichzeitige Einnahme von Cimetidin erreicht werden, da Cimetidin die Bildung des toxischen DADPS-Metaboliten Hydroxylamin inhibiert [45].

\section{Ausblick auf neue Therapieansätze}

Für einige der neueren Immunmodulatoren und Immunsuppressiva, die in den letzten Jahren bei verschiedenen Dermatosen eingesetzt wurden, liegen inzwischen auch Einzelfallberichte zum kutanen LE vor. Demnach zeigt Tacrolimus 0,1\% bei wenig infiltrierten, kutanen LE-Manifestationen eine gewisse positive Wirkung, wohingegen beim CDLE kein Effekt festzustellen war $[30,58,63]$. Tacrolimus ist möglicherweise in der beschriebenen LE-Variante eine therapeutische Alternative zur lokalen Kortikosteroidtherapie.

Mycophenolatmofetil wurde in einzelnen, verschiedenen LE-Varianten als wirksam mitgeteilt. In der Dosierung von $2-3 \mathrm{~g} / \mathrm{d}$ ist Mycophenolatmofetil bei den klassischen, kutanen LE-Varianten, CDLE und SCLE, als wirksam beschrieben. Auch für die selteneren LE-Varianten Chilblain Lupus, LE tumidus und LE profundus wurden in Einzelfallberichten gute Ergebnisse mitgeteilt $[5,21,22,48]$.

Es ist sicher verfrüht, diese neueren Immunmodulatoren/Immunsuppressiva für die LE-Therapie zu empfehlen. Es bleibt abzuwarten, ob sich ihr Einsatz in größeren Patientenkollektiven bewährt.

\section{Literatur}

${ }^{1}$ Atra E, Sato EI. Treatment of cutaneous lesions of systemic lupus erythematosus with thalidomide. Clin Exp Rheumatol 1993; 11: 487-493

2 Belgi G, Friedmann PS. Traditional therapies: glucocorticoids, azathioprine, methotrexate, hydroxyurea. Clin Exp Dermatol 2002; 27: 546-554

${ }^{3}$ Beutner EH, Blazczyk M, Jablonska S, Chorzelski TP, Kumar V, Wolska H. Studies on criteria of the European Academy of Dermatology and Venerology for the classification of cutaneous lupus erythematosus. Int J Dermatol 1991; 30: 411 - 417

${ }^{4}$ Boehm IB, Boehm GA, Bauer R. Management of cutaneous lupus erythematosus with low-dose methotrexate: indication for modulation of inflammatory mechanisms. Rheumatol Int 1999; 18: 59-62

${ }^{5}$ Boehm I, Bieber T. Chilblain lupus erythematosus Hutchinson: successful treatment with mycophenolate mofetil. Arch Dermtol 2001; 137: $235-236$

${ }^{6}$ Bohmeyer J, Achenbach M, Westenberger M, Stadler R. ThalidomidTherapie des kutanen Lupus erythematodes. Hautarzt 2002; 53 : $744-748$

${ }^{7}$ Bottomley WW, Goodfield MJ. Methotrexate for the treatment of discoid lupus erythematosus. Br J Dermatol 1995; 133: 655-656

${ }^{8}$ Burrows NP, Bhogal B, Black M, Rustin M, Ishida-Yamamoto A, Kirtsching $\mathrm{G}$ et al. Bullous eruption of systemic lupus erythematosus: a clinicopathological study of four cases. Br J Dermatol 1993; 20: $128-338$

${ }^{9}$ Calabrese L, Fleischer AB. Thalidomide: current and potential clinical applications. Am J Med 2000; 108: 487-495

${ }^{10}$ Callen JP, Klein J. Subacute cutaneous lupus erythematosus. Clinical serologic, immunogenetic, and therapeutic considerations in seventytwo patients. Arthritis Rheum 1988; 31: 1007 - 1013

${ }^{11}$ Carneiro JR, Sato EI. Double-blind, randomized, placebo controlled trial of methotrexate in systemic lupus erythematosus. J Rheumatol 1999; 26: $1275-1279$

${ }^{12}$ Chocair PR, Duley JA, Simmonds HA, Cameron JS. The importance of thiopurine methyltransferase activity for the use of azathioprine in transplant recipients. Transplantation 1992; 53: 1051 - 1056

${ }^{13}$ Coburn PR, Shuster S. Dapsone and discoid lupus erythematosus. Br J Dermatol 1982; 106: 105-106

14 Drenkard C, Alarcón-Segovia D. The new prognosis of systemic lupus erythematosus: treatment-free remission and decreased mortality and morbidity. Irs Med Assoc J 2000; 2: 382 - 387

${ }^{15}$ Duong DJ, Spigel T, Moxley RT, Gaspari AA. American experience with low-dose thalidomide therapy for severe cutaneous lupus erythematosus. Arch Dermatol 1999; 135: 1079- 1087

${ }^{16}$ Egner W. The use of laboratory tests in the diagnosis of SLE. J Clin Pathol 2000; 53: 424-432

${ }^{17}$ Fenton DA, Black MM. Low-dose dapsone in the treatment of subacute cutaneous lupus erythematosus. Clin Exp Dermatol 1986; 11: $102-103$

18 Gladman DD. Prognosis and treatment of systemic lupus erythematosus. Curr Opin Rheum 1996; 8: 430-437

${ }^{19}$ Goldstein E, Carey W. Discoid lupus erythematosus: successful treatment with oral methotrexate. Arch Dermatol 1994; 130: 938 - 939

${ }^{20}$ Gourley MF, Austin HA, Scott D et al. Methylprednisolone and cyclophosphamide, alone or in combination, in patients with lupus nephritis. A randomized, controlled trial. Ann Intern Med 1996; 125: $549-557$

${ }^{21}$ Goyal S, Nousari HC. Treatment of resistant discoid lupus erythematosus of the palms and soles with mycophenolate mofetil. J Am Acad Dermatol 2001; 45: $142-144$

22 Hanjami HM, Nousari CH. Mycophenolate mofetil for the treatment of cutaneous lupus erythematosus with smoldering systemic involvement. Arch Dermatol 2002; 138: 1616-1617

${ }^{23}$ Healy E, Kieran E, Rogers S. Cutaneous lupus erythematosus-a study of clinical and laboratory prognostic factors in 65 patients. J Med Sci 1995; 164: 113 - 115

${ }^{24}$ Hochberg MC. The epidemiology of systemic lupus erythematosus. In: Wallace DJ, Hahn BH (eds.). Dubois' Lupus Erythematosus. Philadelphia: Lea \& Febiger, 1997: 49-65

${ }^{25}$ Holtmann J, Neustadt D, Klein J, Callen J. Dapsone is an effective therapy for the skin lesions of subacute cutaneous lupus erythematosus 
and urticarial vasculitis in a patient with C2 deficiency. J Rheumatol 1990; 17: $1222-1225$

${ }^{26}$ Housman TS, Jorizzo JL, McCarty MA, Grummer SE, Fleischer AB, Sutej PG. Low-dose thalidomide therapy for refractory cutaneous lesions of cutaneous lupus erythematosus. Arch Dermatol 2003; 139: 50-54

27 Jewell ML, McCauliffe DP. Patients with cutaneous lupus erythematosus who smoke are less responsive to antimalarial treatment. J Am Acad Dermatol 2000; 42: 983-987

28 Johansson-Stephansson E, Koskimies S, Partanen J, Kariniemi AL. Subacute cutaneous lupus erythematosus. Genetic markers and clinical and immunological findings in patients. Arch Dermatol 1989; 125: $791-796$

${ }^{29}$ Jones SK. Ocular toxicity and hydroxychloroquine: guidelines for screening. Br J Dermatol 1999; 140: $3-7$

${ }^{30}$ Kanekura T, Yoshi N, Terasaki K, Miyoshi H, Kanzaki T. Efficacy of topical tacrolimus for treating the malar rash of systemic lupus erythematosus. Br J Dermatol 2003; 148: 353 - 356

${ }^{31}$ Knop J, Bonsmann G, Happle R et al. Thalidomide in the treatment of sixty cases of chronic discoid lupus erythematosus. Br J Dermatol 1983; 108: $461-466$

${ }^{32}$ Kuhn A, Specker C, Ruzicka T, Lehmann P. Methotrexate treatment for refractory subacute cutaneous lupus erythematosus. J Am Acad Dermatol 2002; 46: 600-603

${ }^{33}$ Le Bozec P, La Guyadec T, Crickx B, Grossin M, Belaich S. Chronic lupus erythematosus in lupus disease. Retrospective study in 136 cases. Presse Med 1994; 23: 1598-1602

${ }^{34}$ Lindskov R, Reymann F. Dapsone in the treatment of cutaneous lupus erythematosus. Dermatologica 1986; 172: 214-217

${ }^{35}$ Millard LG, Rowell NR. Abnormal laboratory test results and their relationship to prognosis in discoid lupus erythematosus: A longterm follow-up study of 92 patients. Arch Dermatol 1979; 115: 1055-1058

${ }^{36}$ Moschella SL. Dermatologic overview of lupus erythematosus and its subsets. J Dermatol 1989; 16: 417-428

${ }^{37}$ Naughton MA, Battaglia E, O'Brien S, Walport MJ, Botto M. Identification of thiopurine methyltransferase (TPMT) polymorphisms cannot predict myelosuppression in systemic lupus erythematosus patients taking azathioprine. Rheumatology 1999; 38: 640-644

${ }^{38}$ Nossent HC, Koldingsnes W. Long-term efficacy of azathioprine treatment for proliferative lupus nephritis. Rheumatology 2000; 39: 969-974

39 O'Loughlin S, Schreter A, Jordon R. Chronic urticaria-like lesions in systemic lupus erythematosus. A review of 12 cases. Arch Dermatol 1978; 114: $879-883$

40 Ochonisky S, Verroust J, Bastuji-Garin S et al. Thalidomide neuropathy incidence and clinicoelectrophysiologic findings in 42 patients. Arch Dermatol 1994; 130: 66-69

${ }^{41}$ Ordi-Ros J, Cortes F, Cucurull E, Mauri M, Bajan S, Vilardelli M. Thalidomide in the treatment of cutaneous lupus refractory to conventional therapy. J Rheumatol 2000; 27: 1429-1433

42 Orfanos CE, Garbe C (Hrsg). Therapie der Hautkrankheiten. Berlin, Heidelberg, New York: Springer-Verlag, 2002: 513-535

43 Parodi A, Caproni M, Cardinali C, Bernacchi E, Fuligni A, De Panfilis G Zane C, Papini M, Veller FC, Vaccaro M, Fabbri P. Clinical, histological and immunopathological features of 58 patients with subacute cutaneous lupus erythematosus. Dermatology 2000; 200: 6-10

${ }^{44}$ Parodi A, Massone C, Cacciapuoti M, Aragone MG, Bondavalli P, Cattarini G, Rebora A. Measuring the activity of the disease in patients with cutaneous lupus erythematosus. Br J Dermatol 2000; 142: 457-460

45 Rhodes LE, Tingle MD, Park BK, Chu P, Verbov JL, Friedmann PS. Cimetidine improves the therapeutic/toxic ratio of dapsone in patients on chronic dapsone therapy. Br J Dermatol 1995; 132: 257-262
${ }^{46}$ Ruzicka T, Meurer M, Braun-Falco O. Treatment of cutaneous lupus erythematosus with etretinate. Acta Derm Venereol 1985; 65: $324-329$

${ }^{47}$ Ruzicka T, Sommerburg C, Goerz G, Kind P, Mensing H. Treatment of cutaneous lupus erythematosus with acitretin and hydroxychloroquine. Br J Dermatol 1992; 127: 513 - 518

${ }^{48}$ Schanz S, Ulmer A, Rassner G, Fierlbeck G. Successful treatment of subacute cutaneous lupus erythematosus with mycophenolate mofetil. Br J Dermatol 2002; 147: 174- 178

${ }^{49}$ Shornick JK, Formica N, Parke AL. Isotretinoin for refractory lupus erythematosus. J Am Acad Dermatol 1991; 24: 49-52

${ }^{50}$ Sontheimer RD. Subacute cutaneous lupus erythematosus. A decade's perspective. Med Clin N Am 1989; 73: 1073 - 1090

51 Stevens RJ, Andujar C, Edwards CJ, Ames PRJ, Barwick AR, Khamashta MA, Hughes GRV. Thalidomide in the treatment of the cutaneous manifestations of lupus erythematosus: experience in sixteen consecutive patients. $\mathrm{Br}$ J Rheumatol 1997; 36: 353 - 359

52 Tan EM, Cohen AS, Fries JF, Masi AT, McShane DJ, Rothfield NF, Schaller JG, Talal N, Winchester RJ. The 1982 revised criteria for the classification of systemic lupus erythematosus. Arthritis Rheum 1982; 25: $1271-1277$

53 Tebbe B, Mansmann U, Wollina U, Auer-Grumbach P, Licht-Mbalyohere A, Arensmeier M, Orfanos CE. Markers in cutaneous lupus erythematosus indicating systemic involvement. A multicenter study on 296 patients. Acta Derm Venereol 1997; 77: 305-308

54 Tebbe B, Orfanos CE. Lupus erythematodes der Haut. Eine Analyse von 97 Patienten. Z Hautkr 1987; 62: 1563-1584

55 Tseng P, Pak G, Washenik K, Pomeranz MK, Shupack JL. Rediscovering thalidomide: a review of its mechanism of acion, side effects, and potential uses. J Am Acad Dermatol 1996; 35: 969-979

56 Tsufsui K, Imai T, Hatta N, Takata M, Takehara K. Widespread pruritic plaques in a patient with subacute cutaneous lupus erythematosus and hypocomplementemia: response to dapsone. J Am Acad Dermatol 1996; 35: 313-315

57 Walcher M, Meurer M, Plewig G, Messer G. Clinical and immunological parameters during thalidomide treatment of lupus erythematosus. Int J Dermatol 2000; 39: 383-388

${ }^{58}$ Walker SL, Kirby B, Chalmers RJG. The effect of topical tacrolimus on severe recalcitrant chronic discoid lupus erythematosus. Br J Dermatol 2002; 147: 405 - 406

${ }^{59}$ Wallace DJ. Antimalarial therapy. In: Wallace DJ, Hannahs Hahn B (eds). Dubois' Lupus erythematosus. Baltimore: Williams \& Wilkins, 1997: $1117-1139$

${ }^{60}$ Watanabe T, Tsuchida T. Classification of lupus erythematosus based upon cutaneous manifestations. Dermatology 1995; 190: 277-283

61 Wozel G. Dapson - Pharmakologie, Wirkungsmechanismus und klinischer Einsatz in der Dermatologie. Dermatol Monatsschr 1993; 179 : $1-9$

62 Wozniacka A, Carter A, McCauliffe DP. Antimalarials in cutaneous lupus erythematosus: mechanisms of therapeutic benefit. Lupus 2002; 11: $71-81$

63 Yoshimasu T, Ohtani T, Sakamoto T, Oshima A, Furukawa F. Topical FK506 (tacrolimus) therapy for facial erythematous lesions of cutaneous lupus erythematosus and dermatomyositis. Eur J Dermatol 2002; 12: $50-52$

${ }^{64}$ Zečević RD, Vojvodić D, Ristić B, Pavlović MD, Stefanović D, Karadaglić D. Skin lesions - an indicator of disease activity in systemic lupus erythematosus? Lupus 2001; 10: $364-367$ 